\title{
Generation of electron spin polarization in disordered organic semiconductors
}

\author{
A. I. Shushin \\ Institute of Chemical Physics, Russian Academy of Sciences, \\ 117977, GSP-1, Kosygin str. 4, Moscow, Russia
}

\begin{abstract}
The generation mechanisms of electron spin polarization (ESP) of charge carriers (electrons and holes, called "doublets") in doublet-doublet recombination and triplet-doublet quenching in disordered organic semiconductors are analyzed in detail. The ESP is assumed to result from quantum transitions between the states of the spin Hamiltonian of the pair of interacting particles. The value of the ESP is essentially determined by the mechanism of relative motion of particles. In our work we have considered the cage and free diffusion models. The effect of possible attractive spin-independent interactions between particles is also analyzed. Estimation with obtained formulas shows that the proposed mechanisms can lead to a fairly strong ESP much larger than the thermal one (at room temperatures).
\end{abstract}

PACS numbers: 73.50.-h, 73.43.Qt, 75.47.-m, 72.25.Dc

\section{INTRODUCTION}

Electron spin polarization (ESP) of charge carriers (electrons and holes) in solid-state systems is the important phenomenon, which is observed in ferromagnetic metals and inorganic semiconductors and result from strong magnetic interactions in these solids $\underset{1,2}{2}$ The ESP effect is very interesting from general physical point of view and important for applications ${ }^{1}-\underline{3}$

As for organic semiconductors (OSCs), magnetic interactions in them are typically fairly weak and no strong ESP is expected. The ESP can, nevertheless, be injected from ferromagnetic solids. ${ }^{3}$ In many experiments the OSCs are applied as spacers (between ferromagnetic leads), in which one can manipulate the ESP by applying electric and magnetic fields. 2,3 The dynamics of electron spins in OSCs is studied quite thoroughly, $, 2,3$ but the majority of works concern the analysis of spin evolution of polarized charge carriers injected into OSCs from polarizing leads (see, for example, refs. [4-10], as well as [2,3] and references therein).

This work concerns the discussion of the mechanisms of ESP generation directly in OSCs (in the absence of spin injection). The possibility of this effect is usually ignored because of above-mentioned weakness of magnetic interactions in these materials. The analysis shows, however, that some spin selective processes in OSCs can result in fairly strong ESP (much larger than the thermal one). Such processes are similar to those, which determine the magnetic field and spin effects (MFEs) in spin selective reactions of paramagnetic particles [usually doublets $(D)$ and triplets $(T)]$

In our investigation we will consider two types of processes, doublet-doublet $(D-D)$ recombination 14,15 and triplet-doublet $(T-D)$ quenching, ${ }^{16-18}$ in which the ESP generation is expected to be very efficient. The ESP results from quantum transitions between the terms of the spin Hamiltonian of the pair of particles $(D-D$ or $T-D)$, assisted by their stochastic relative motion. The terms are determined by the Zeeman and spin exchange interactions and transitions are induced by the hyperfine (HFI), anisotropic part of the Zeeman (AZI), or zero field splitting (ZFS) interactions, depending on the process considered. The strong dependence of the exchange interaction on the interparticle distance $r$ leads to the significant localization of transitions in space and essential dependence of the ESP generation efficiency on the characteristic properties of relative motion.

Noteworthy is that the theory of diffusion assisted ESP generation in liquid phase reactions has already been developed. ${ }^{17-21}$ It is based on the stochastic Liouville equation (SLE) approach, 22,23 which allows one to rigorously describe the generation of MFEs, (including the ESP). As applied to similar processes in OSCs, however, this SLE-based theory needs certain modifications, due to some specific features of interaction and relative motion of paramagnetic particles in these materials.

The effect of these specific features on the ESP in OSCs is considered within two models of relative motion of particles: cage and free diffusion. Both models are shown to predict similar expressions for the ESP both in geminate and bulk processes. The proposed theory enables one to take into account possible spin-independent (i.e. Coulomb) interaction $U(r)$ between reacting particles, which can strongly affect the MFEs ${ }^{24,25}$ Simple estimations demonstrate that the attractive interaction $U(r)$ results in significant increase of the ESP. The evaluation of the ESP for realistic values of parameters of the theory shows that the considered mechanisms can be very efficient leading to a quite large ESP in OSCs.

\section{GENERAL FORMULATION}

The mechanisms of the ESP generation in diffusion assisted liquid pase spin-selective reactions are thoroughly investigated in the theory of MFEs. ${ }^{17-21}$ In our work we will briefly analyze most important results, but will be mainly concerned with extending and adapting them to the processes under study.

We consider two types of spin-selective processes: 
the doublet-doublet $(D-D)$ recombination and tripletdoublet $(T-D)$ quenching, in which doublet particles are associated with electrons or holes $(D=e, h)$. The most part of the consideration concerns geminate reactions, though bulk processes are also discussed.

The kinetic scheme the geminate variant of both processes can be represented as

$$
X_{a}+X_{b} \stackrel{w_{d}}{\longleftarrow}\left[X_{a} \ldots X_{b}\right] \stackrel{\hat{K}_{R_{2}}}{\longrightarrow} X_{R}, \quad(X=D, T) .
$$

Here $X_{a}$ and $X_{b}$ denote $D$ - or $T$-particles involved in the reaction. The reaction kinetics is essentially controlled by the rate $w_{d}$ of dissociation of the coupled state (cage) $\left[X_{a} \ldots X_{b}\right]$ and spin selective reaction rate $\hat{K}_{R}$.

The kinetics of ESP generation in these processes is described with the spin density matrix $\rho(t)$ of pairs of paramagnetic particles $X_{a}$ and $X_{b}$. Naturally, $\rho(t)$ depends on the interparticle coordinate $\mathbf{r}$. For simplicity, we assume that the precess (2.1) is isotropic, i.e. the interparticle interactions and initial condition depend on the distance $r=|\mathbf{r}|$ between particles. In this case $\rho$ depends only on $r: \rho(\mathbf{r}, t) \equiv \rho(r, t)$

The spin evolution of the pairs is governed by the spin Hamiltonian $H(r, t)$, which can, in general, be time dependent. The explicit form of $H(r, t)$ will be specified below for both considered processes.

In the above-formulated assumptions the space/time evolution of the pair density matrix $\rho(r, t)$ is determined by the $\operatorname{SLE}(\hbar=1)$

$$
\dot{\rho}=-i[H, \rho]-\left(\hat{K}_{R}+\hat{L}\right) \rho,
$$

with $[H, \rho]=H \rho-\rho H$. In this SLE the operator $\hat{L}$ describes the relative motion of particles, which is treated as hopping migration and is characterized by the average jump length $\lambda$ and the rate of jumps $w_{h}$. The term $\hat{K}_{R} \rho$ represents the effect spin selective reaction:

$$
\hat{K}_{R} \rho=\frac{1}{2} \kappa_{R}\left(P_{R} \rho+\rho P_{R}\right)
$$

where $P_{R}$ is the operator of projection onto the reactive states of the pair and $\kappa_{R}$ is the reaction rate. In general, $\kappa_{R} \equiv \kappa_{R}(r)$ depends on the distance $r$ and this dependence $\kappa_{R}(r)$ is strongly localized at $r$ small distances.

The ESP essentially depends on the initial spin and spatial state. In general, the initial density matrix can be written as

$$
\rho(t=0)=p_{0}(r) \rho_{0},
$$

where $p_{0}(r)$ is the initial spatial distribution (see below) and $\rho_{0}$ is the initial spin density matrix.

The observable under study is the ESP $\mathcal{P}(t \rightarrow \infty)$ of $D$-particles. For brevity of notations, in what follow we will mainly analyzed the absolute value of the ESP $\mathcal{P}$. As for the ESP sign, it can easily be obtained, as it is demonstrated in Sec. V.

In our work we consider the most important mechanisms of the ESP generation in two above-mentioned processes and within cage and free diffusion models of relative motion of particles:

1) Cage model. In the cage model the considered process is represented by the kinetic scheme (2.1), in which $w_{d}$ is the rate of monomolecular dissociation of the cage and $\hat{K}_{R}$ is the rate operator of spin-selective reaction, independent of the distance $r\left(\kappa_{R}\right.$ is independent of $\left.r\right)$.

The operator $\hat{L}$, essentially controlling the kinetics of the process [see eq. (2.3)], is defined by

$$
\hat{L} \rho=w_{d} \rho .
$$

Detailed analysis shows 18 that non-adiabatic transitions between terms of the Hamiltonian $H$, which determine the ESP generation, are localized near the distance $d$ of closest approach (of reacting particles) in the narrow region of width $\delta r \sim \alpha^{-1} \ll d$, where $\alpha^{-1}$ is the size of the exchange interaction (see below). The distance $d$ is, in turn, expected to be of order of the hopping length $\lambda$. In such a case, it is quite reasonable to assume, that the cage size $r_{c} \sim \lambda(\sim d)$, and associate the cage dissociation rate $w_{d}$ with the rate $w_{h}$ of jumps: $w_{d} \sim w_{h}$.

For pairs in these cages (of volume $\mathcal{V}_{c} \sim \lambda^{3}$ ) the relative $X_{a}-X_{b}$ coordinate $\mathbf{r}$ is suggested to be randomly (homogeneously) distributed. Note that for the homogeneous distribution over $\mathbf{r}$ the initial condition for the SLE can be written in the form (2.4): $\rho(t=0)=p_{0} \rho_{0}$ with $p_{0}=\mathcal{V}_{c}^{-1}$.

In the cage model, for example, the ESP of $D$-particles, escaped from the cage, can be evaluated by formula

$$
\begin{aligned}
\mathcal{P} & =2 w_{d}\left|\int_{0}^{\infty} d t\left\langle\operatorname{Tr}\left[S_{D_{z}} \rho(r, t)\right]\right\rangle_{\mathbf{r} \in \mathcal{V}_{c}}\right| \\
& =2 w_{d}\left|\left\langle\operatorname{Tr}\left[S_{D_{z}} \widetilde{\rho}(r, \epsilon=0)\right]\right\rangle_{\mathbf{r} \in \mathcal{V}_{c}}\right|,
\end{aligned}
$$

where $S_{D_{z}}$ is the $z$-projection (along the magnetic field B) of the $D$-particle spin $\mathbf{S}_{D}, \tilde{\rho}(\epsilon)=\int_{0}^{\infty} d t e^{-\epsilon t} \rho(t)$ is the Laplace transform of $\rho(t)$ and

$$
\langle\rho\rangle_{\mathbf{r} \in \mathcal{V}_{c}}=\mathcal{V}_{c}^{-1} \int_{\mathbf{r} \in \mathcal{V}_{c}} d^{3} r \rho(r)
$$

is the average over the homogeneous distribution in the cage (of volume $\mathcal{V}_{c}$ ).

2) Free diffusion model. In the free diffusion model the considered processes can, formally, be expressed by the same scheme (2.1), in which, however, the reaction and dissociation kinetics is non-exponential and controlled by relative diffusion of reacting particles. In this model

$$
\hat{L} \rho=-D_{p} r^{-1} \nabla_{r}^{2}(r \rho) \text { and } \kappa_{R}=\kappa_{r} \delta(r-d),
$$

where $D_{p}=D_{X_{a}}+D_{X_{b}},\left(D_{p} \sim \lambda^{2} w_{h}\right)$, is the coefficient of relative diffusion of particles $X_{a}$ and $X_{b}, \nabla_{r}=\partial / \partial r$, and $\hat{\kappa}_{R}$ is defined by $\hat{\kappa}_{R}=\frac{1}{2} \kappa_{r}\left(P_{R} \rho+\rho P_{R}\right)$ [eq. (2.3)]. In the free diffusion model the SLE should be solved with the reflective inner boundary condition at the distance of closest approach $d:\left.\stackrel{18}{=} \nabla_{r} \rho(r, t)\right|_{r=d}=0$, and the outer condition $\rho(r \rightarrow \infty, t) \rightarrow 0$. 
The ESP of $D$-particle is expressed by formula 18,19

$$
\mathcal{P}=2\left|\int_{r>d} d^{3} r \operatorname{Tr}\left[S_{D_{z}} \rho(r, t \rightarrow \infty)\right]\right|
$$

The kinetics of ESP generation in the free diffusion model has already been analyzed in detail for both $D-D$ and $T-D$ processes $^{17-21}$ that is why in what follows we will restrict ourselves to brief summary of some results of this analysis interesting for our work.

\section{III. $D-D$ RECOMBINATION}

The $D-D$-recombination is described by the kinetic scheme (2.8) with $X_{a}=D_{a}$ and $X_{b}=D_{b}$, in which $\hat{K}_{R}$ is the operator of reaction in the singlet $(S)$ state of the $D_{a}-D_{b}$ pair, given by eq. (2.3) with $P_{R}=P_{S}=|S\rangle\langle S|$.

The most important mechanism of ESP generation in this type of reactions is known as $S T_{-}$-mechanism. ${ }^{14,15}$ Here we will consider the specific features of this mechanism in organic semiconductors.

\section{A. Interactions}

The spin Hamiltonian of the $D_{a}-D_{b}$ pair is assumed to be represented as a sum

$$
H(r, t)=H_{0}(r)+V(t)
$$

where $H_{0}(r)$ and $V(t)$ are the steady state and randomly distributed (and may be fluctuating) parts, respectively.

The steady state part of the Hamiltonian

$$
H_{0}(r)=H_{z}+H_{e x}(r)
$$

is diagonal in the basis of the total electron spin $\hat{\mathbf{S}}=$ $\hat{\mathbf{S}}_{a}+\hat{\mathbf{S}}_{b}$ (called $S T$-basis) and consists of the Zeeman interaction (in the external magnetic field $\mathbf{B}$ )

$$
H_{z}=\Omega_{0}\left(S_{a_{z}}+S_{b_{z}}\right)
$$

in which $\Omega_{0}=\bar{g} \beta B$ is the Zeeman splitting (we assume that the isotropic parts of $g$-factors of both particles are the same: $\bar{g}_{a}=\bar{g}_{b}=\bar{g}=2$ ), and the exchange interaction

$$
H_{e x}=J(r)\left(\frac{1}{2}+2 \mathbf{S}_{a} \mathbf{S}_{b}\right)
$$

exponentially decreasing with $r$ :

$$
J(r)=J_{0} \exp [-\alpha(r-d)],\left(J_{0}>0\right),
$$

where $d$ is the distance of closest approach: $d \gg \alpha^{-1}$.

In the $S T_{-}$-mechanism the ESP is assumed to result from quantum transitions between $U_{S}(r)$ and $U_{T_{ \pm}}(r)$ terms of $\hat{H}_{0}(r)$ near the $S T_{-}$-crossing region at $r=r_{t}$ (see Fig. 1). These transitions are caused by the fluctuating part $V(t)$ of the Hamiltonian (non-diagonal in the $S T$-basis), which is a sum of two terms:

$$
V(t)=V_{H}(t)+V_{Z}(t) .
$$

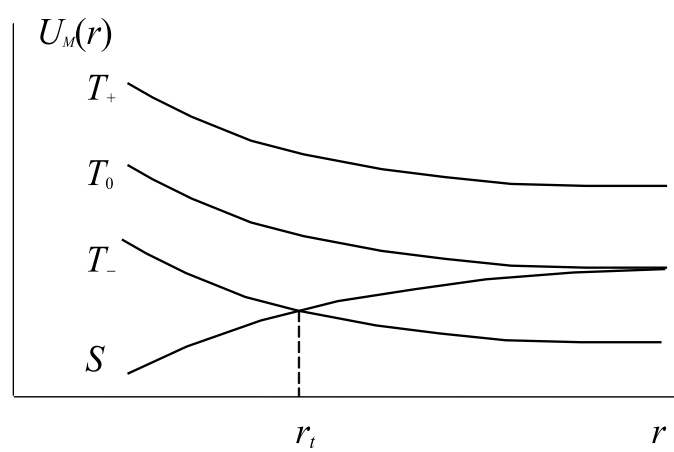

FIG. 1. The schematic picture of terms $U_{M}(r),(M=$ $\left.S, T_{0}, T_{ \pm}\right)$, of the spin Hamiltonian $H_{0}(r)$ [eq. (3.2)] of the $D-D$ pair (for $J_{0}>0$ ), in which $r_{t}$ is the coordinate of the region of efficient quantum transitions.

The first term $V_{H}(t)$ represents the hyperfine interaction (HFI), which in the considered realistic case of a large number of nuclei can quite accurately be treated as the interaction of electron spins $\mathbf{S}_{a}$ and $\mathbf{S}_{b}$ with (classical) random magnetic fields $\mathbf{B}_{a}$ and $\mathbf{B}_{b}$, respectively:

$$
V_{H}(t)=\bar{g} \beta\left[\mathbf{S}_{a} \mathbf{B}_{a}(t)+\mathbf{S}_{b} \mathbf{B}_{b}(t)\right] .
$$

Distributions of fields are $\mathbf{B}_{a}$ and $\mathbf{B}_{b}$ isotropic, and Gaussian with $\left\langle\mathbf{B}_{\nu}\right\rangle=0$ and mean squares $\left\langle B_{\nu}^{2}\right\rangle \sim$ $\sum_{j} I_{\nu_{j}}\left(1+I_{\nu_{j}}\right) a_{\nu_{j}}^{2}, \quad(\nu=a, b)$, determined by hyperfine coupling constants $a_{\nu_{j}}{ }^{26}$ Hopping of $D_{a}$ and $D_{b}$ particles results in sudden changing of nuclear magnetic fields, which can be considered as stochastic vectors $\mathbf{B}_{\nu}(t), \quad(\nu=a, b)$. For simplicity, the correlation functions of projections $B_{\nu_{q}}(t),(q=x, y, z)$ are taken in the form $\left\langle B_{\nu_{q}}(t) B_{\nu_{q^{\prime}}}(0)\right\rangle \sim \delta_{q q^{\prime}}\left\langle B_{\nu}^{2}\right\rangle \exp \left(-w_{H_{\nu}} t\right.$ ) (so that $\left.\left\langle V_{H}(t) V_{H}(0)\right\rangle \sim e^{-w_{H_{\nu}}{ }^{t}}\right)$.

The second term $V_{Z}(t)$ in eq. (3.6) is the anisotropic part of the Zeeman interaction (AZI):

$$
V_{z}(t)=\beta\left[\mathbf{S}_{a} \hat{g}_{a}^{\prime}(t) \mathbf{B}+\mathbf{S}_{b} \hat{g}_{b}^{\prime}(t) \mathbf{B}\right]
$$

where

$$
\hat{g}_{\nu}^{\prime}=\hat{g}_{\nu}-\bar{g}_{\nu} \text { with } \bar{g}_{\nu}=\operatorname{Tr}\left(\hat{g}_{\nu}\right), \quad(\nu=a, b) .
$$

The representation (3.8) implies that $\operatorname{Tr}\left(\hat{g}_{\nu}^{\prime}\right)=0$. As in the case of the HFI the time dependence $\hat{g}_{\nu}^{\prime}(t)$ results from $D$-particle hopping. We will use the simple form of the correlation function $27\left\langle g_{\nu_{q q^{\prime}}}^{\prime}(t) g_{\nu_{q^{\prime} q}}^{\prime}(0)\right\rangle \sim$ $e^{-w_{Z_{\nu}} t}, \quad\left(q \neq q^{\prime}\right), \quad\left(\right.$ which means that $\left\langle V_{Z}(t) V_{Z}(0)\right\rangle \sim$ $\left.e^{-w_{H_{\nu}}{ }^{t}}\right)$

\section{B. ESP generation kinetics}

In order to evaluate the $\operatorname{ESP} \mathcal{P}$ we need to specify the initial spin state $\rho_{0}$ [see eq. (2.4)]. In our analysis, for 
certainty, we consider the equilibrium (isotropic) state:

$$
\rho_{0}=\frac{1}{4}\left(P_{S}+P_{T}\right),
$$

where $P_{S}=|S\rangle\langle S|$ and $P_{T}=\sum_{m=0, \pm}\left|T_{m}\right\rangle\left\langle T_{m}\right|$. Note, that for other initial spin states the ESP is, actually, proportional to that for $\rho_{0}$ (3.10) ${ }^{25}$

\section{Cage model}

We have already noted that quantum transitions, resulting in the ESP, are localized in the narrow region of width $\delta r \sim \alpha^{-1} \ll d$ (recall that $d \sim \lambda$ ). In such a case the ESP generation can properly be described by treating spin evolution of $D-D$ pairs in all positions of them within this region as cages, whose dissociation rate is associated with the hopping rate $w_{h}$ (see Sec. II): $w_{d} \lesssim w_{h}$.

The interaction $V$ in thus introduced cages should be considered as time independent though randomly distributed, since the fluctuations of $V(t)$ are formed just as a result of hopping motion, i.e. at times $t \gtrsim \tau_{h}=w_{h}^{-1}$.

Under the assumption of homogeneous distribution of $D-D$ coordinate $\mathbf{r}$ in the cage (see Sec. II) and with the use of eq. (2.6) one obtains the following formula for the ESP $\mathcal{P}$, generated in $D-D$ recombination,

$$
\mathcal{P}=\left|\mathcal{P}_{-}-\mathcal{P}_{+}\right|=\left|\left\langle p_{-+}(r)\right\rangle_{\mathbf{r} \in \mathcal{V}_{c}}\right|,
$$

where $\mathcal{P}_{ \pm}=w_{d}\left\langle\widetilde{\rho}_{ \pm}(r)\right\rangle_{\mathbf{r} \in \mathcal{V}_{c}}$ are the contributions of $T_{ \pm} \leftrightarrow$ $S$ transitions, in which $\widetilde{\rho}_{ \pm}^{c}(r)=\left\langle T_{ \pm}|\widetilde{\rho}(r, \epsilon=0)| T_{ \pm}\right\rangle$, and $p_{-+}(r)=w_{d}\left[\widetilde{\rho}_{-}(r)-\widetilde{\rho}_{+}(r)\right]$.

Solution of the SLE in the second order in the randomly distributed interaction $V$ yields formula

$$
p_{-+}(r)=\bar{p}\left\langle\left|V_{ \pm}\right|^{2}\right\rangle\left[\frac{1}{w_{c}^{2}+\Omega_{-}^{2}(r)}-\frac{1}{w_{c}^{2}+\Omega_{+}^{2}(r)}\right]
$$

with $\left|V_{ \pm}\right|=\left|\left\langle S|V| T_{+}\right\rangle\right|=\left|\left\langle S|V| T_{-}\right\rangle\right|, \quad \Omega_{ \pm}(r)=\Omega_{0} \pm$ $2 J(r), w_{c}=w_{d}+\frac{1}{2} \kappa_{R}$ being the $S-T$-dephasing rate in the cage, and $\bar{p}=\frac{1}{2}\left(\kappa_{R} w_{c}\right) /\left[w_{d}\left(w_{d}+\kappa_{R}\right)\right]$.

Substituting this formula into eq. (3.11) one can obtain the following expression for the $\mathrm{SPE} \mathcal{P}$, corresponding to the initial equilibrium spin state (3.10):

$$
\mathcal{P}=p_{c}\left|\varphi_{\omega_{-}}-\varphi_{\omega_{+}}\right|\left[\omega_{c} /\left(1+\omega_{c}^{2}\right)\right],
$$

in which $\varphi_{\omega_{ \pm}}=\arctan \left(\omega_{ \pm}\right)+\left(2 \omega_{c}\right)^{-1} \ln \left(1+\omega_{ \pm}^{2}\right)$ with $\omega_{ \pm}=\left(\Omega_{0} \pm 2 J_{0}\right) / w_{c}$,

$$
\omega_{c}=\Omega_{0} / w_{c} \text { and } p_{c} \approx \frac{1}{2} p_{R_{c}}\left(\frac{\left\langle\left|V_{ \pm}\right|^{2}\right\rangle}{w_{c} w_{d}}\right)\left(\frac{4 \pi d^{2}}{\alpha \mathcal{V}_{c}}\right) .
$$

Here $p_{R c}=\kappa_{R} /\left(w_{d}+\kappa_{R}\right)$ is the probability of reaction in $S$-state and $\mathcal{V}_{c}$ is the volume of the cage.

Note that in the presence of $V(t)$-fluctuations the rate formulas (3.13) and (3.14) are still valid but $w_{c}=w_{d}+$ $\frac{1}{2} \kappa_{R}+w_{\mu},(\mu=H, Z)$, where $w_{\mu}$ is the rate of decay of correlation function $\left\langle V_{\mu}(t) V_{\mu}(0)\right\rangle$, introduced above.
It also is worth noting that the ESP for any other initial spin state is closely related to that (3.13) for the equilibrium state $\rho_{E}(3.10)$. For example, for the triplet initial state $\rho_{0}=\rho_{T}=\frac{1}{3} P_{T}$ the $\operatorname{ESP} \mathcal{P}\left(\rho_{T}\right)=(4 / 3) \mathcal{P}\left(\rho_{E}\right) / p_{R_{C}}$.

Formula (3.13) is fairly cumbersome. Fortunately, in applications it is quite sufficient to use simpler limiting expressions at large $J_{0}>\Omega_{0}, w_{c}$ and small $J_{0}<\Omega_{0}, w_{c}$ :

$$
\begin{aligned}
& \mathcal{P}=\mathcal{P}_{s}=\left(\pi p_{c}\right) \frac{\omega_{c}}{1+\omega_{c}^{2}} \quad \text { for } J_{0}>\Omega_{0}, w_{c} \\
& \mathcal{P}=\mathcal{P}_{w}=8 p_{c}\left(\frac{J_{0}}{w_{c}}\right) \frac{\omega_{c}}{\left(1+\omega_{c}^{2}\right)^{2}} \quad \text { for } J_{0}<\Omega_{0}, w_{c} .
\end{aligned}
$$

It is easily seen that the considered two cases $J_{0}>\Omega_{0}$ and $J_{0}<\Omega_{0}$ correspond $r_{t}>d$ and $r_{t}<d$, respectively, where $r_{t}$ is the distance of $S T_{-}$crossing (see Fig. 1).

\section{Free diffusion model}

Simple formulas for the ESP have also been derived in the free diffusion model ${ }^{17-21}$ Naturally, the ESP, generated in diffusion controlled $D-D$ recombination, depends (but weakly) on the initial distance $r_{i}$. Below, for certainty, we present the formulas in the realistic case $r_{i} \approx d$.

The most interesting expressions for the ESP at large $J_{0}>\Omega_{0}, w_{f}$ and small $J_{0}<\Omega_{0}, w_{f}\left[w_{f}\right.$ is the correlation rate of $V(t)$ fluctuations (see below)] turn out to be similar to those obtained above in the cage model: ${ }^{17-21}$

$$
\begin{array}{ll}
\mathcal{P}=\mathcal{P}_{s}=\left(\pi p_{f}\right) \frac{\omega_{f}}{1+\omega_{f}^{2}} \quad \text { for } J_{0}>\Omega_{0}, w_{f}, \\
\mathcal{P}=\mathcal{P}_{w}=8 p_{f}\left(\frac{J_{0}}{w_{f}}\right) \frac{\omega_{f}}{\left(1+\omega_{f}^{2}\right)^{2}} & \text { for } J_{0}<\Omega_{0}, w_{f} .
\end{array}
$$

Here

$$
\omega_{f}=\Omega_{0} / w_{f} \text { and } p_{f} \approx \frac{1}{2} p_{R_{f}}\left(\frac{\left\langle\left|V_{ \pm}\right|^{2}\right\rangle}{w_{f}^{2}}\right)\left(\frac{d w_{f}}{\alpha D_{p}}\right)
$$

with $w_{f}=w_{\mu},(\mu=H, Z)$, being the decay rate of the correlation function $\left\langle V_{\mu}(t) V_{\mu}(0)\right\rangle$ (defined in Sec. IIIA).

Similarly to the cage model $p_{R_{f}}=l_{S} / r_{i} \approx l_{S} / d$ is the probability of (diffusion controlled) reaction in $S$ state with the rate $\kappa_{R}$, given by eq. (2.8), in which $l_{S}=$ $d\left(\kappa_{r} d / D_{p}\right)\left[1+\left(\kappa_{r} d / D_{p}\right)\right]^{-1}$ is the reaction radius.

\section{Some general properties of $D-D$ ESP}

The important specific features of the ESP for different limits and mechanisms (discussed above) are conveniently represented in terms of the dimensionless functions $\Phi_{\mu}^{\chi}(\omega)$, in which the superscript $\mu$ specifies the ESP mechanism (see Sec. IIIA): HFI $(\mu=H)$ and AZI $(\mu=Z)$, while the subscript $\chi$ indicates the value of $J_{0}$ : large $(\chi=s)$ and small $(\chi=w)$. In accordance with above-obtained expressions, one can write

$$
\mathcal{P}=p_{R_{\gamma}}\left\langle v_{\mu}^{2}\right\rangle \bar{P}_{\chi} \Phi_{\mu}^{\chi}\left(\omega_{\gamma}\right) .
$$


Here $p_{R_{\gamma}}$ and $\omega_{\gamma}$ are defined in eqs. (3.14) and (3.19) for the cage $(\gamma=c)$ and free diffusion $(\gamma=f)$ models, respectively. The parameters $\left\langle v_{\mu}^{2}\right\rangle$ are the dimensionless squares of interactions defined by formulas

$$
\left\langle v_{H}^{2}\right\rangle=(\bar{g} \beta)^{2} \frac{\left\langle B_{a}^{2}\right\rangle+\left\langle B_{b}^{2}\right\rangle}{12 w_{\gamma}^{2}},\left\langle v_{z}^{2}\right\rangle=\frac{g_{a}^{\prime}: g_{a}^{\prime}+g_{b}^{\prime}: g_{b}^{\prime}}{40 \bar{g}},
$$

with $w_{\gamma},(\gamma=c, f)$, given in eqs. (3.14) and (3.19).

a. Strong exchange interaction limit $(\chi=s)$. In the limit of strong exchange interactions (large $J_{0}$ ) we get for the HFI $(\mu=H)$ and AZI $(\mu=Z)$ mechanisms

$$
\Phi_{H}^{s}(\omega)=\omega /\left(1+\omega^{2}\right), \quad \Phi_{Z}^{s}(\omega)=\omega^{3} /\left(1+\omega^{2}\right),
$$

where the parameter $\bar{P}_{s}$ is written for the cage $(\gamma=c)$ and free diffusion $(\gamma=f)$ models as follows:

$$
\begin{array}{ll}
\bar{P}_{s}=\left(\frac{1}{2} \pi\right)\left(w_{c} / w_{d}\right)\left(4 \pi d^{2} / \alpha \mathcal{V}_{c}\right) & \text { for } \gamma=c, \\
\bar{P}_{s}=\left(\frac{1}{2} \pi\right)\left(d w_{f} / \alpha D_{p}\right) & \text { for } \gamma=f .
\end{array}
$$

b. Weak exchange interaction limit $(\chi=w)$. In the weak exchange interaction limit (small $J_{0}$ ) one obtains

$$
\Phi_{H}^{w}(\omega)=\omega /\left(1+\omega^{2}\right)^{2}, \quad \Phi_{z}^{w}(\omega)=\omega^{3} /\left(1+\omega^{2}\right)^{2},
$$

with

$$
\bar{P}_{w}=(8 / \pi)\left(J_{0} / w_{\gamma}\right) \bar{P}_{s},
$$

both in the cage and free diffusion models (for $\gamma=c, f$ ).

The functions $\Phi_{\mu}^{\chi}(\omega)$ are shown in Fig. 2. Notice that all dependences $\Phi_{\mu}^{\chi}(\omega)$, except $\Phi_{z}^{s}(\omega)$, are non-monotonic with maximum at $\omega \sim 1$.

Note that in the free diffusion model the formulas for the ESP are derived under the assumption of not very high mobilities of particles (fairly small diffusion coefficients) so that $\xi_{\Omega}=\Omega_{0} d^{2} / D_{p}>1 \stackrel{18}{\underline{10}}$ In the opposite limit $\xi_{\Omega}=\Omega_{0} d^{2} / D_{p}<1$ the ESP is essentially determined by the parameter $\xi_{w}=w_{c} d^{2} / D_{p}$. For $\xi_{w}>1$ we get the results presented above independently of the value of $\xi_{\Omega}$. For $\xi_{w} \ll 1$, however, the behavior of $\Phi_{\mu}^{\chi}(\omega)$ at $\xi_{\omega}<1$ becomes non-analytic: for example, $\Phi_{H}^{s}(\omega) \sim \omega^{-3 / 2} \underline{18}$

\section{T-D QUENCHING}

The $T-D$ quenching is another example of spin selective processes, leading to the generation of the ESP of $D$-particles. The kinetic scheme of the geminate $T-D$ process can be represented in the form (2.1) with $X_{a}=T$ and $X_{b}=D$ (in OSCs $D$-particle is associated with trapped electron).

The quenching is believed ${ }^{28}$ to result in the transition to $X_{R}=\left(S_{0} D^{*}\right)_{D}$, where $S_{0}$ is the molecule in the ground (singlet) state, and $D^{*}$ is the excited doublet (free electron), i.e. the quenching rate is expected to be non-zero only in the doublet $\left(\left|D_{ \pm 1 / 2}\right\rangle\right)$ states of $T-D$ pair, corresponding to the total spin $S=1 / 2$. In this case the

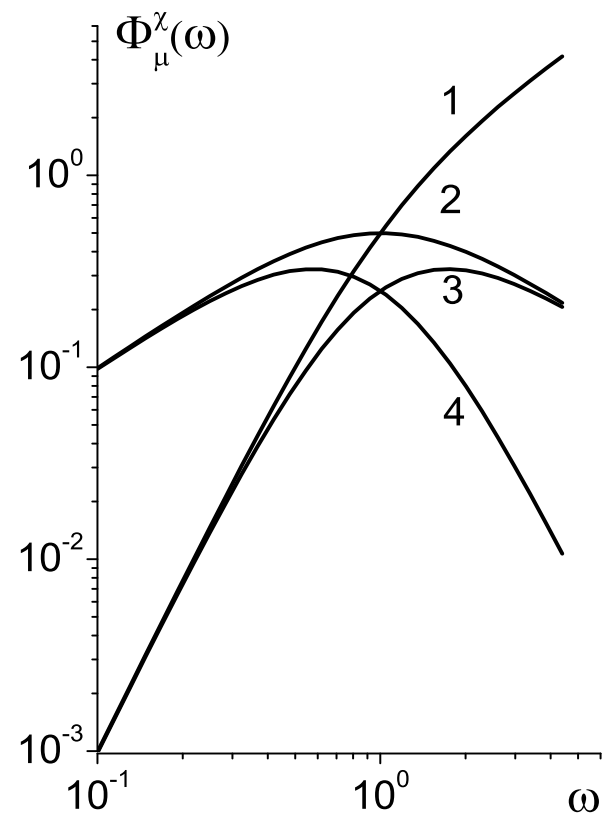

FIG. 2. The functions $\Phi_{\mu}^{\chi}(\omega)$, which determine the ESP dependence on the Zeeman splitting $\omega=\Omega_{0} / w_{\gamma}$ in cage $(\gamma=c)$ and free diffusion $(\gamma=c)$ models [see eqs. (3.22) and (3.25)]: (1) $\Phi_{Z}^{s}(\omega),(2) \Phi_{H}^{s}(\omega),(3) \Phi_{Z}^{w}(\omega)$, and (4) $\Phi_{H}^{w}(\omega)$ [see eqs. (3.22) and [3.25]. In these functions the superscript $\chi$ and subscript $\mu$ specify the exchange interaction strength and the mechanism of the ESP generation, respectively, $(\chi=s, w$ and $\mu=H, Z)$.

quenching operator $\hat{K}_{R}$ is represented in the form (2.3) with $P_{R}=P_{D}=\left|D_{+1 / 2}\right\rangle\left\langle D_{+1 / 2}|+| D_{-1 / 2}\right\rangle\left\langle D_{-1 / 2}\right|$.

It is clear from the above comments to the general scheme (2.1) that, in principle, there are two types of $D$-particles involved in the process under study: $D$ and $D^{*}$ [escaped from the reaction $(D)$ and resulting from reaction $\left(D^{*}\right)$ ]. In OSCs these particles are associated with trapped electrons and electrons in in the conducting band, respectively $\underline{28}$ The $T-D$ quenching gives rise to the ESP of both particles. In the analysis, however, we will assume that the kinetic and MFE parameters of these types of $D$-particles are different, i.e. the contributions of them to the ESP are experimentally distinguishable.

In our work we will concentrate on the evaluation of the ESP of $D^{*}$ particles.

The methods of estimating ESP of both $D$ particles for liquid phase processes, i.e. in the diffusion model of relative motion of particles, have already been discussed in a number of papers $\frac{17,20,21}{}$ Below we will analyze some specific features of the ESP generation as applied to disordered semiconductors, considering more thoroughly the cage model, as an example. 


\section{A. Interactions}

In our analysis we assume that the spin Hamiltonian of the $T-D$ pair (similar to that for $D-D$ systems) can be represented as a sum $H(r, t)=H_{0}(r)+V(t)$, where $H_{0}(r)$ and $V(t)$ are the steady state and randomly distributed (and may be fluctuating) parts, respectively.

The steady state part

$$
H_{0}(r)=H_{z}+H_{e x}(r),
$$

diagonal in the basis of the total electron spin $\mathbf{S}=\mathbf{S}_{T}+$ $\mathbf{S}_{D}$, is a sum of the Zeeman and exchange interactions:

$$
H_{z}=\omega_{0} S_{z} \text { and } H_{e x}=J(r)\left(\frac{1}{2}+2 \mathbf{S}_{T} \mathbf{S}_{D}\right),
$$

in which $S_{z}=S_{T z}+S_{D_{z}}, \omega_{0}=g \beta B$ (we assume that $g_{D}=g_{T}=g=2$ ), and $J(r)$ is given by eq. (3.5). The terms $U_{M}(r),\left(M=Q_{m}, D_{m}\right)$, of the Hamiltonian $H_{0}(r)$ are displayed in Fig. 3.

Transitions in the regions of crossing of $U_{M}(r)$-terms are determined by the fluctuating zero-field-splitting (ZFS) interaction

$$
V=V_{T}=D_{T}\left(S_{T_{z}^{\prime}}^{2}-\frac{1}{3} S_{T}^{2}\right)+E_{T}\left(S_{T_{x}^{\prime}}^{2}-S_{T_{y}^{\prime}}^{2}\right),
$$

where $S_{T_{j}},\left(j=x^{\prime}, y^{\prime}, z^{\prime}\right)$, are the projections of the spin of $T$-exciton on the eigenaxes of the ZFS tensor. Usually $D_{T} \gg E_{T}$, that is why below we will neglect the terms $\sim E_{T}$, taking $V_{T} \approx D_{T} S_{T_{z}^{\prime}}^{2}$. The fluctuations result from hopping motion of $T$ exciton over sites with randomly oriented directions of axes $x^{\prime}, y^{\prime}, z^{\prime}$. These fluctuations show themselves in those of interaction matrix elements $V_{T_{M M^{\prime}}}(t) \equiv\left\langle M\left|V_{T}(t)\right| M^{\prime}\right\rangle$, correlation function of which is assumed to be of the form $27\left\langle V_{T_{M M^{\prime}}}^{*}(t) V_{T_{M M^{\prime}}}(0)\right\rangle_{\Omega^{\prime}}=$ $\left\langle\left|V_{M M^{\prime}}^{2}\right|\right\rangle e^{-w_{T} t}$ (here $M, M^{\prime}=Q_{m}, D_{m}$ and $\langle\ldots\rangle_{\Omega^{\prime}}$ denotes averaging over orientations of the ZFS eigenaxes).

\section{B. ESP generation kinetics}

In $T-D$ system the ESP is assumed to result from quantum transitions in the regions of crossing of terms $U_{Q_{ \pm 3 / 2}}(r)$ and $U_{Q_{ \pm 1 / 2}}(r)$ with reactive terms $U_{D_{ \pm 1 / 2}}(r)$. These regions are indicated in Fig. 3.

Similarly to the $D-D$ ESP, the ESP in $T-D$ quenching will be evaluated for the equilibrium initial spin state

$$
\rho_{0}=\rho_{E}=\frac{1}{6}\left(P_{D}+P_{Q}\right),
$$

where $P_{D}$ and $P_{Q}$ are the operators of projection onto the subspaces of $D$ - and $Q$-states, respectively: $P_{D}=$ $\sum_{m= \pm 1 / 2}\left|D_{m}\right\rangle\left\langle D_{m}\right|$ and $P_{Q}=\sum_{m= \pm 1 / 2, \pm 3 / 2}\left|Q_{m}\right\rangle\left\langle Q_{m}\right|$. The ESP for other initial spin state are, actually, proportional to that for $\rho_{E} \cdot 25$

In the majority of $T-D$ processes the above-mentioned ZFS-induced quantum transitions can be calculated perturbatively in the second order in $V$. In this approximation the effect of transitions is represented as a sum of

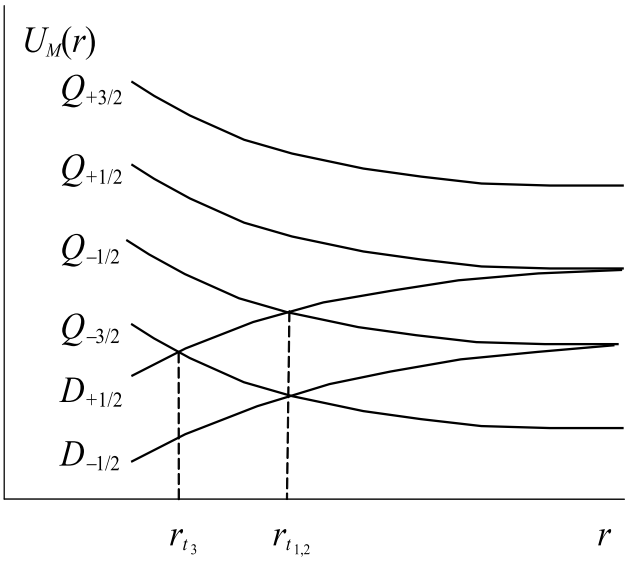

FIG. 3. The schematic picture of terms $U_{M}(r),(M=$ $\left.D_{ \pm 1 / 2}, Q_{ \pm 1 / 2}, Q_{ \pm 3 / 2}\right)$, of the spin Hamiltonian $H_{0}(r)$ [eq. [4.1] ] of the $T-D$ pair (for $J_{0}>0$ ), in which $r_{t_{1,2}}$ and $r_{t_{3}}$ are the coordinates of the regions of efficient quantum transitions.

contributions of all pairs of coupled terms, which can be evaluated with formulas derived above for the $D-D$ ESP $\mathcal{P} .18$ It is worth noting that these formulas represent the effect of quantum transitions on the $D-D$ ESP of escaped articles. However, similar expressions are, certainly, valid for the product particles as well. 21

Analysis of quantum transitions between terms (displayed in Fig. 3) shows that the contributions to the ESP $\mathcal{P}_{*}$ of the $D^{*}$-particle can be represented as a sum of three contributions of type of (3.12):

$$
\mathcal{P}=\mathcal{P}_{*}=\frac{2}{3}\left|\delta \mathcal{P}_{1 / 2,3 / 2}+\delta \mathcal{P}_{1 / 2,1 / 2}+\delta \mathcal{P}_{1 / 2,-3 / 2}\right|,
$$

in which $\delta \mathcal{P}_{\mu, \mu^{\prime}}$ are defined by

$$
\delta \mathcal{P}_{m, m^{\prime}}=\mathcal{P}_{D_{-m} Q_{-m^{\prime}}}-\mathcal{P}_{D_{m} Q_{m^{\prime}}},
$$

[with $m=1 / 2, m^{\prime}=1 / 2,3 / 2$ ], where $\mathcal{P}_{D_{m} Q_{m^{\prime}}}$ is the contribution of the transition $D_{m} \leftarrow Q_{m^{\prime}}$.

\section{Evaluation of ESP in the cage model}

In the cage model we can obtain the expression for $\delta \mathcal{P}_{m, m^{\prime}}$ in terms of the distance dependent solution $p_{m m^{\prime}}(r)$ similar to that derived above [see eq. (3.12)]

$$
\delta \mathcal{P}_{m, m^{\prime}}=\left\langle p_{m m^{\prime}}(r)\right\rangle_{\mathbf{r} \in \mathcal{V}_{c}} .
$$

In this formula

$$
p_{m m^{\prime}}(r)=\bar{p}\left\langle\left|V_{m m^{\prime}}^{2},\right|\right\rangle\left[\frac{1}{w_{c}^{2}+\Omega_{-}^{2}(r)}-\frac{1}{w_{c}^{2}+\Omega_{+}^{2}(r)}\right],
$$

where the parameter $\bar{p}=\frac{1}{2}\left(\kappa_{R} w_{c}\right) /\left[w_{d}\left(w_{d}+\kappa_{R}\right)\right]$ is identical to that in eq. (3.12), and

$$
\Omega_{ \pm}(r)=\alpha_{m m^{\prime}} \Omega_{0} \pm 2 J(r)
$$


with $\alpha_{ \pm 1 / 2, \pm 3 / 2}=\alpha_{ \pm 1 / 2, \mp 1 / 2}=1$ and $\alpha_{ \pm 1 / 2, \mp 3 / 2}=2$. As for $\left\langle\left|V_{m m^{\prime}}^{2}\right|\right\rangle$, for $m$ and $m^{\prime}$, corresponding to the contributions (4.6), they are written as $\left\langle\left|V_{ \pm 1 / 2, \pm 3 / 2}^{2}\right|\right\rangle=$ $\frac{1}{45} D_{T}^{2},\left\langle\left|V_{ \pm 1 / 2, \mp 1 / 2}^{2}\right|\right\rangle=\frac{1}{15} D_{T}^{2}$, and $\left\langle\left|V_{ \pm 1 / 2, \mp 3 / 2}^{2}\right|\right\rangle=\frac{4}{45} D_{T}^{2}$.

Note that similar to the $D-D$ process in the presence of $V_{T}$-fluctuations the dephasing rate is written as $w_{c}=$ $w_{d}+\frac{1}{2} \kappa_{R}+w_{T}$ [rather than as that defined in eq. (3.20)], where $w_{T}$ is the decay rate of the correlation function $\left\langle V_{T_{M M^{\prime}}}^{*}(t) V_{T_{M M^{\prime}}}(0)\right\rangle_{\Omega^{\prime}}$, defined in Sec IVA.

Substitution of all terms (4.8) into eq. (4.5) yields the final expression for the ESP of $D^{*}$ (see below).

\section{Combined formula for ESP, predicted by two models}

The $T-D$ ESP, predicted in the above-analyzed cage model and previously studied free diffusion model, $17,20,21$ can be represented in one combined expression similar to that (3.20) obtained for the $D-D$ ESP

$$
\mathcal{P}_{*}=\frac{2}{3} p_{R_{\gamma}}\left\langle v_{T}^{2}\right\rangle \bar{P}_{\chi}\left[\Phi_{H}^{\chi}\left(\omega_{\gamma}\right)+2 \Phi_{H}^{\chi}\left(2 \omega_{\gamma}\right)\right],(\chi=s, w) .
$$

In this expression $\left\langle v_{T}^{2}\right\rangle=\frac{2}{45}\left(D_{T}^{2} / w_{c}^{2}\right)$, the functions $\Phi_{H}^{\chi}\left(\omega_{\gamma}\right)$ are defined in eqs. (3.22) and (3.25), and the parameters $\bar{P}_{\chi}$ are given in eqs. (3.23), (3.24), and (3.26).

The difference between the cage and free diffusion ESP lies in the parameters $p_{R_{\gamma}}$ and $\omega_{\gamma}$ : for the cage $\operatorname{ESP}(\gamma=$ c) $p_{R_{c}}=\kappa_{R} /\left(w_{d}+\kappa_{R}\right)$ and $\omega_{c}=\Omega_{0} / w_{c}$ [see eq. (3.14)], while for free diffusion $\operatorname{ESP}(\gamma=f) p_{R_{f}}=\left(\kappa_{r} d / D_{p}\right) /[1+$ $\left.\left(\kappa_{r} d / D_{p}\right)\right]$ and $\omega_{f}=\Omega_{0} / w_{f}$ with $w_{f}=w_{T}$ [eq. (3.19)].

It is worth noting that the dependence of $\mathcal{P}_{*}$ on $\omega_{\gamma}$, (i.e. on $\Omega_{0}$ ), predicted by both the cage and free diffusion models, is determined by that of $\Phi_{H}^{\chi}(\omega)$, i.e. function $\mathcal{P}_{*}\left(\omega_{\gamma}\right)$ is non-monotonic the maximum of $\mathcal{P}_{*}\left(\omega_{\gamma}\right)$ is located at $\omega_{\gamma}=\omega_{\gamma_{m}} \sim 1$ (see Fig. 2).

\section{DISCUSSION}

The obtained formulas allow us to analyze easily the specific features of the ESP in $D-D$ and $T-D$ processes.

\section{A. General remarks}

Before estimating the ESP some remarks are needed on the validity of models and approximations applied.

1. So far in our work we have discussed the absolute value $\mathcal{P}$ of the ESP. As for the ESP sign, it can be characterized by the sign factor $S_{\mathcal{P}}= \pm 1$, defined by the relation $2\left\langle S_{D z}\right\rangle=S_{\mathcal{P}} \mathcal{P}$, in which the average $\langle\ldots\rangle$ is made over spin state and distances (see Sec. II). The factor $S_{\mathcal{P}}$ can easily be obtained from the qualitative analysis of quantum transitions in $D-D$ and $T-D$ systems. In general, $S_{\mathcal{p}}$ is essentially determined by the sign of the exchange interaction $S_{\mathcal{P}} \sim \operatorname{sign}\left(J_{0}\right)$. Below we will briefly discuss the ESP sign in the most realistic case $J_{0}>0$ (for $J_{0}>0$ the terms are shown in Figs. 1 and 3).

a) In the $D-D$ process the ESP of survived $D$-particles is determined by $S T_{-}$-transitions, leading to the decrease of the population of $T_{-}$-state, i.e. to $S_{\mathcal{P}}=+1$.

b) In the $T-D$ quenching the ESP of $D^{*}$ particles results from quantum transitions, associated with contributions (4.6) resulting in larger population of $D_{+1 / 2}$-state (because of stronger coupling between initial $Q$ states and this $D$-state). Therefore for $D^{*}$ particles $S_{\mathcal{P}}=+1$ as well.

2. In the proposed theory the $\operatorname{ESP} \mathcal{P}$ is evaluated in the second order in $\zeta_{\gamma}=\left\langle\left|V^{2}\right|\right\rangle / w_{\gamma} \ll 1,(\gamma=c, f)$, which implies that $\mathcal{P} \ll 1$. This does not mean, however, that the generated ESP is small, in general. For example, the effect of attractive interparticle interactions can lead to strong increase of the ESP (see Sec. V.B.2).

3 . In the cage model the spin selective reaction rate $\kappa_{R}$ is suggested to be independent of the distance $r$. This assumption is, in principle, not quite consistent taking into account that $J(r)$ is treated as distance dependent. Notice, that the dependence $\kappa_{R}$ on $r$ could be analyzed within generalized formulas [see eqs. (3.12) and (3.12)], though, the modification of formulas would result in the strong complication of the expression for $\mathcal{P}$. To justify the simplifying assumption on $\kappa_{R}$ independence of $r$ it is worth noting that the manifestation of $\kappa_{R}(r)$-dependence in $\mathcal{P}$ is fairly weak. It is especially weak in two aboveconsidered limits of strong and weak exchange interaction, most important for further applications.

a) For strong exchange interactions the quantum transitions, determining the ESP, are highly localized in the regions of term crossing (Figs 1, 3). The distance dependence $\kappa_{R}(r)$ in these regions can be neglected.

b) In the weak exchange interaction limit the dependence of $\mathcal{P}$ on the rate $\kappa_{R}$ results from that of the probability of reaction in the cage $p_{R_{\gamma}} \sim \kappa_{R},(\gamma=c, f)$. In the free diffusion model the weak effect of the $r$-dependence of reactivity is demonstrated in earlier works, ${ }^{17-21}$ Here we will discuss the cage model, in which this effect shows itself in the average over $\mathbf{r}$ in formula (3.11) for $\mathcal{P}$, i.e. in the average $\left\langle p_{R_{c}} J\right\rangle_{r}$, where $p_{R_{c}}=\kappa_{R} /\left(w_{d}+\kappa_{R}\right)$. It is seen that in $\left\langle p_{R_{c}} J\right\rangle_{r}$ the $\kappa_{R}(r)$ dependence manifests itself only weakly. For example, in the low reactivity limit, when $p_{R_{c}} \approx \kappa_{R} / w_{d}$ and the manifestation is strongest, the dependence $\kappa_{R}(r) \sim e^{-\alpha r}$, identical to that of $J(r)$ [see eq. (3.5)], results only in two-fold reduction of the ESP. This effect is too small to be of any importance for our semiquantitative study of the problem.

\section{B. Estimations of the ESP}

\section{Bulk processes}

In our analysis we have considered the ESP generation in geminate processes. It can be known, however, that the geminate $\operatorname{ESP} \mathcal{P}$, calculated for the equilibrium initial spin state, is directly related to the rate $\mathcal{K}$ of ESP 
generation in the corresponding bulk processes: ${ }^{25}$

$$
\mathcal{K}=k_{0} \mathcal{P} \text { with } k_{0}=4 \pi r_{c} D_{p}
$$

(note that for $T-D$ system $\mathcal{P} \equiv \mathcal{P}_{*}$ ). In this formula $D_{p}$ is the coefficient of relative diffusion and $r_{c}$ is the radius of the cage $\left(r_{c} \sim \lambda\right)$ in the cage model, or the radius of efficient quantum transitions $\left(r_{c} \sim d\right)$ in the free diffusion model. Our assumption that $d \sim \lambda$ (Sec. II) means that in both models the estimations for $r_{c}$ are close.

Note that the parameter $r_{c}$, whose meaning in the diffusion theory is clear, $, 17,18,20,21$ is not quite well defined in the cage model. This uncertainty of the cage-model definition of $r_{c}$ is, however, not very strong and is not essential for our semiquantitative analysis.

Sometimes it is convenient to relate the rate of ESP generation to the reaction rate:

$$
\mathcal{K}_{r} \approx\left(p_{s} p_{R_{\gamma}}\right) k_{0}, \quad(\gamma=c, f),
$$

where $p_{R \gamma}$ is the probability of reaction in the reactive state [see eqs. (3.14) and (3.19)] and $p_{s}$ is statistical factor, representing the relative number of reactive spin states: for $D-D$ and $T-D$ pairs we get $p_{s}=1 / 4$ and $p_{s}=1 / 3$, respectively. In eq. (5.2) we neglected small contribution of quantum transitions to the reaction rate.

Formulas (5.1) and (5.2) are fairly suitable for studying the efficiency of the ESP generation in different types of bulk processes. The explicit expressions for the ESP are, naturally, determined by the particular kinetic scheme of the process, and should be discussed as applied to the interpretation of specific experiments.

\section{Effect of attractive interparticle interaction}

The proposed theory is based on the assumption that particles $X_{a}$ and $X_{a}$ [see eq. (2.1)] undergo free stochastic motion. In reality, however, the ESP can be strongly affected by the interparticle interaction $U(r)=k_{B} T u(r)$.

Especially strong effect of the interaction is expected in electron-hole $(e-h)$ recombination, kinetics of which is known to be markedly influenced by the Coulomb $e-h$ interaction. Significant interaction effect can also be observed in some $T-D$ pairs, in which $D$-particle is the electron $(e)$ or hole $(h) \underline{6}$ For these pairs $U(r) \sim-\frac{1}{2} \alpha_{T} E^{2}(r)$, where $\alpha_{T}$ is a polarizability of $T$ state and $E(r)=e /\left(\varepsilon r^{2}\right)$ is the strength of the Coulomb field of $e$ (or $h$ ) particle in the semiconductor with dielectric constant $\varepsilon$.

In the presence of the well-type attractive potential $U(r)$ the ESP $\mathcal{P}_{c}$ can approximately be estimated within the simple kinetic scheme, taking into account the effect of the cage within the well,$\stackrel{24,25}{2}$ which hereafter is denoted as $U$-cage. The size $R_{c}$ of the $U$-cage, determined by the relation $\left|U\left(R_{c}\right)\right|=k_{B} T$, is expected to be significantly larger than the size $r_{c}$ of the primary cage, considered above in the cage model: $R_{c} \gg r_{c} \sim \lambda$.

The kinetic scheme of the process in the $U$-cage (in the well) can also be represented as (2.1), but with the reaction $\left(\mathcal{W}_{r}\right)$ and dissociation $\left(\mathcal{W}_{d}\right)$ rates $\underline{24,25}$

$$
\mathcal{W}_{r}=\mathcal{K}_{r} / \mathcal{V}_{U} \text { and } \mathcal{W}_{d}=\left(K_{0} / \mathcal{V}_{U}\right) e^{-u_{a}},
$$

in which $u_{a}=U_{a} /\left(k_{B} T\right)$ is activation energy of dissociation (the well depth), $K_{0}=4 \pi R_{c} D_{p}$ is the bimolecular rate of capture into the well, and $\mathcal{V}_{U}$ is the volume of the $U$-cage. The volume can be estimated as $\mathcal{V}_{U} \approx(4 \pi / 3) R_{U}^{3}$, where $R_{U}$ is the radius of the thermal distribution within the well, i.e. $U\left(R_{U}\right) \approx U_{b}+k_{B} T$, with $U_{b}=-U_{a}$ being the energy of the bottom of the well.

In the $U$-cage the ESP generation is described by the rate

$$
\mathcal{W}_{c}=\mathcal{P} \mathcal{W}_{0}, \text { where } \mathcal{W}_{0}=k_{0} / \mathcal{V}_{U}
$$

The kinetic scheme (2.1) predicts exponential kinetics of the decay of $U$-cage population, but with different decay rates for different spin states of pairs. In particular, for non-reactive states ( $T$ and $Q$ states for $D-D$ and $T-D$ pairs, respectively), whose depopulation kinetics determine the ESP generation, the decay rate is $\mathcal{W}_{d}$. In such a case the $U$-cage affected ESP $\mathcal{P}_{c}$ is written as

$$
\mathcal{P}_{c}=\mathcal{P}\left(\mathcal{W}_{0} / \mathcal{W}_{d}\right)=\mathcal{P}\left(r_{c} / R_{c}\right) e^{u_{a}}
$$

(recall that for $T-D$ system $\mathcal{P} \equiv \mathcal{P}_{*}$ ). Similarly the $U$ cage affected ESP generation rate $\mathcal{K}_{c}$ is given by $\mathcal{K}_{c}=$ $\mathcal{P}_{c} K_{0}=\mathcal{K} e^{u_{a}}$. As for the decay rate of reactive states, it is equal to $\mathcal{W}_{r}+\mathcal{W}_{d}$ and, therefore, the $U$-cage affected reaction rate is represented as $\mathcal{K}_{r_{c}}=\left[\mathcal{W}_{r} /\left(\mathcal{W}_{r}+\mathcal{W}_{d}\right)\right] K_{c}$.

It is seen from eq. (5.5) that the attractive interparticle interaction can result in the strong increase of the ESP.

Noteworthy is that formulas (5.5) are obtained for not very deep wells, when $\eta=\mathcal{W}_{c} / \mathcal{W}_{d}<1$ and one can neglect the contribution of quantum transitions to the reaction rate $\mathcal{W}_{r}$. To find $\mathcal{P}_{c}$ and $\mathcal{K}_{c}$ in the opposite limit $\eta>1$, one has to solve complicated kinetic equations, in general. Some simple estimations can, nevertheless, be made in the realistic case of strong $J(r)$, large Zeeman splitting $\omega_{\gamma}>1$, and high reactivity (when $p_{R_{\gamma}} \approx 1$ ). In this case only quantum transitions between non-reactive and reactive states, localized in crossing regions, contribute the ESP. These contributions can be easily evaluated for both $D-D$ and $T-D$ reactions:

a) For $D-D$ system only $T_{-} \rightarrow S$ transitions contribute to the ESP so that $\mathcal{P}_{c}$ can be written as

$$
\mathcal{P}_{c}=\frac{1}{4} \mathcal{W}_{S T_{-}} /\left(\mathcal{W}_{d}+\mathcal{W}_{S T_{-}}\right),
$$

where $\mathcal{W}_{S T_{-}}=\mathcal{W}_{S \leftarrow T_{-}}=4 \mathcal{P} \mathcal{W}_{0}$ is the transition rate, whose value is obtained by comparing the expression (5.6) with eq. (5.5) in the limit $\mathcal{W}_{S T_{-}} \ll \mathcal{W}_{d}$.

b) For $T-D$ system similar analysis leads to formula

$$
\begin{aligned}
\mathcal{P}_{c} \equiv \mathcal{P}_{*_{c}} & =\frac{1}{6}\left[\mathcal{W}_{2} /\left(\mathcal{W}_{d}+\mathcal{W}_{2}\right)+\mathcal{W}_{-} /\left(\mathcal{W}_{d}+\mathcal{W}_{+}\right)\right] \\
& =\left(\mathcal{P}_{*} \mathcal{W}_{0}\right) /\left[\mathcal{W}_{d}+(9 / 2) \mathcal{P}_{*} \mathcal{W}_{0}\right]
\end{aligned}
$$

in which $\mathcal{W}_{ \pm}=\mathcal{W}_{3} \pm \mathcal{W}_{1}$, with $\mathcal{W}_{1} \equiv \mathcal{W}_{D_{-1 / 2} \leftarrow Q_{-3 / 2}}=$ $\frac{3}{2} \mathcal{P}_{*} \mathcal{W}_{0}$ and $\mathcal{W}_{3} \equiv \mathcal{W}_{D_{+1 / 2} \leftarrow Q_{-3 / 2}}=2 \mathcal{W}_{1}$, and $\mathcal{W}_{2} \equiv$ 
$\mathcal{W}_{D_{+1 / 2} \leftarrow Q_{-1 / 2}}=3 \mathcal{W}_{1}$. Similarly to the case of $D-D$ system the expression of the rates $\mathcal{W}_{j},(j=1-3)$, in terms of the ESP $\mathcal{P} \equiv \mathcal{P}_{*}$ is found by comparison of eq. (5.7) with eq. (5.5) (for $\mathcal{P}_{c} \equiv \mathcal{P}_{*_{c}}$ ) at $\mathcal{W}_{1} \ll \mathcal{W}_{d}$.

Formulas (5.6) and (5.7), looking quite natural in the diffusion model, need some comments as applied to the cage model. These expression imply that within the primary cage (at $r \lesssim r_{c}$ ) a large variety of relative positions $r$ of particles, including those in the term-crossing region, are accessible. Note that possible migration of particles over these positions can result in $V(t)$-fluctuations and, thus, to some change of $w_{c}$ (see Sec. IIIB.1 and IVB.1). However, in the considered case of strong $J(r)$ and large $\omega_{\gamma}$ this change does not lead to any change of the ESP 18,20,21 Noteworthy is also that the abovementioned large variety of $r$ in primary cages can be realized as a result of reencounters of reacting particles at different points in the $U$-cage. In such a case the ESP is quite properly described by the original eqs. (5.6), (5.7).

\section{Magnitude of the ESP}

As we have already noted above, the dependence of the $D-D$ ESP $\mathcal{P}\left(\omega_{\gamma}\right) \sim \Phi_{\mu}^{\chi}\left(\omega_{\gamma}\right)$ on the dimensionless Zeeman splitting $\omega_{\gamma}=\Omega_{0} / w_{\gamma},(\gamma=c, f)$, is non-monotonic for both HFI and AZI mechanisms of ESP generation (see Sec. IIIA) and both models of relative motion: the cage $(\gamma=c)$ and free diffusion $(\gamma=f)$ [except the case $\mu=Z, \chi=s$ [3.22)]. Similar non-monotonic behavior of $\mathcal{P}_{*}\left(\omega_{\gamma}\right)$ is observed in $T-D$-quenching [eq. [4.10)]. In all these cases the maximum value $\mathcal{P}_{m}=\mathcal{P}\left(\omega_{\gamma_{m}}\right)$ [or $\mathcal{P}_{m}=\mathcal{P}_{*}\left(\omega_{\gamma_{m}}\right)$ for $T-D$ process] is located at $\omega_{\gamma_{m}} \sim 1$.

Such a behavior of $\mathcal{P}\left(\omega_{\gamma}\right)$ allows one to simplify the analysis of the ESP by restricting the discussion to maximal values $\mathcal{P}_{m}$ of the effect. In what follows, for certainty, we will estimate $\mathcal{P}$ at $\omega_{\gamma}=1$, for which $\Phi_{\mu}^{\chi}(1) \sim$ $\left[\Phi_{H}^{\chi}(1)+2 \Phi_{H}^{\chi}(2)\right] \sim 1$, so that the maximal values $\mathcal{P}_{m}$ for $D-D$ and $\stackrel{H}{T-D}$ processes can be written as

$$
\mathcal{P}_{m} \sim p_{R_{\gamma}}\left\langle v_{\mu}^{2}\right\rangle \bar{P}_{\chi}, \quad(\mu=H, Z, T \text { and } \chi=s, w),
$$

In this formula $p_{R_{\gamma}}$ is the probability of reaction in reactive spin states [see eqs. (3.14) and (3.19)]. The parameter $\left\langle v_{\mu}^{2}\right\rangle$ is the dimensionless coupling of terms of $H_{0}(r)$ [eqs. (3.21) and (4.10). As for $\bar{P}_{\chi}$, it describes the dependence of the ESP on the strength of $J(r)(\chi=s$ and $\chi=w$ for strong and weak $J$, respectively). Formulas for $\bar{P}_{\chi}$, different in two considered models of motion, are presented in eqs. (3.23), (3.24) and (3.26).

Of certain interest is the above-mentioned case of AZI mechanism $(\mu=Z)$ and strong $J(r)(\chi=s)$, in which $\Phi_{Z}^{s}(\omega)$ is a monotonically increasing function. Below it will be analyzed more thoroughly. Here we only note that in this case at large $\omega \sim B$ the ESP can be very large.

Now we will estimate the parameters in eq. (5.8):

1. The largest ESP is, naturally, expected in the case of strong reactivity resulting in a high reaction probability
$p_{R_{\gamma}}$. In what follows we will assume that $p_{R_{\gamma}} \sim 1$.

2. The parameter $\left\langle v_{\mu}^{2}\right\rangle(\mu=H, Z, T)$ is suggested to be small: $\left\langle v_{\mu}^{2}\right\rangle<1$, to ensure the validity of the applied perturbation approximation (in $V$ ). Noteworthy is that for AZI mechanism $(\mu=Z)$ the validity of this approximation implies also not very large value of the magnetic field $B$ (to be sure that the value of $\left\langle V_{z}^{2}\right\rangle / w_{c}^{2}$ is small).

3. The parameter $\bar{P}_{\chi}$ [as it follows from eqs. (3.23), (3.24) and [3.26) ] is smaller than 1 , but is not very small. The fact is that, in reality, $w_{e} \sim w_{d} \sim w_{f}$ and $D_{p} \sim$ $\lambda^{2} / w_{d} \sim d^{2} / w_{d}$, so that the small value of $\bar{P}_{\chi}$ results from the relation $\bar{P}_{\chi} \sim(\alpha d)^{-1}$, in which $\alpha d \lesssim 10$.

Combining the estimations of these three parameters we can obtain the ESP, but to calculate it more accurately we need to specify the process under study.

For example, let us consider the cage model prediction for the ESP $\mathcal{P} \equiv \mathcal{P}_{*}$ of $D^{*}$ particles in $T-D$ quenching. The value of the parameter $\left\langle v_{T}^{2}\right\rangle$ for the $T-D$ system is determined by the ZFS constant $D_{T}$ and $w_{c} \sim w_{h}$. For realistic values $D_{T}=3 \cdot 10^{9} \mathrm{~s}^{-1}$ and $w_{h}=10^{9} \mathrm{~s}^{-1}$ one obtains $\left\langle v_{T}^{2}\right\rangle=\frac{2}{45}\left(D_{T}^{2} / w_{c}^{2}\right) \approx 0.4$. Taking into account the above estimations $p_{R_{\gamma}} \sim 1$ and $(\alpha d)^{-1} \gtrsim 0.1$ we get (for $\omega_{c}=1$ ) the value $\mathcal{P}_{*_{m}} \gtrsim 4 \cdot 10^{-2}$, much larger than the thermal ESP $\mathcal{P}_{*_{t h}} \approx 1.4 \cdot 10^{-3}$ (at room temperature).

This value of $\mathcal{P}_{*_{m}}$ is obtained in the case of strong exchange interaction $(\chi=s)$, predicting largest ESP. Of course, in the opposite limit $(\chi=w)$ the ESP is smaller with the factor $J_{0} / w_{\gamma} \ll 1$ [see eq. (3.26)].

The attractive $T-D$ interaction can result in the strong increase of the $U$-cage affected ESP $\mathcal{P}_{*_{c}}: \mathcal{P}_{*_{c}} \sim \mathcal{P}_{*} e^{u_{a}}$, where $u_{a}$ is the depth of the potential well ( $U$-cage), as it follows from eq. (5.5). In particular, even in the case of fairly shallow attractive well of the interaction potential, corresponding to $u_{a}=2.0\left(k_{B} T\right)$, this interaction gives rise to the significant (about one order of magnitude) increase of the ESP $\mathcal{P}_{*_{e}}$. Taking into account the aboveobtained estimation of $\mathcal{P}_{*}$, we expect, that the interaction affected $T-D$ ESP can be fairly large: $\mathcal{P}_{*_{e}} \sim 0.1$.

Similar estimations can also be made for the $D-D$ ESP. Because of this similarity we are not going to do them here, but restrict ourselves to some comments on the AZI mechanism of the ESP $(\mu=Z)$. The fact is that values of $\left\langle v_{z}^{2}\right\rangle$ [see eq. (3.21)] typical for organic semiconductors are very small: $\left\langle v_{z}^{2}\right\rangle \lesssim 10^{-5}$ [due to small $g^{\prime}: g^{\prime}$ [3.8)], and for not very strong magnetic fields $B$, corresponding to $\omega_{\gamma} \lesssim 10^{3}$, the ESP is fairly small: $\mathcal{P} \lesssim 10^{-3}$. It can be significantly larger, however, in the presence of the attractive interaction ( $U$-cage), as it follows from above relations. Note also, that in some organic semiconductors, doped with heavy atoms, the Zeeman interaction can be strongly anisotropic $\frac{12}{2}$ resulting in fairly large $\left\langle v_{z}^{2}\right\rangle \gtrsim 10^{-4}$ and, thus, in the ESP much larger than the thermal one.

Concluding our analysis of the $U$-cage effect on the ESP, we recall that the applied formulas (5.3) and (5.5) are valid for relatively shallow wells, when $\eta=$ $\left(\mathcal{P} \mathcal{W}_{0}\right) / \mathcal{W}_{d}<1$. For deeper wells, for which $\eta>1$, a 
fairly large $U$-cage effect is expected, though the calculation of the ESP in this limit is a rather complicated problem, in general. Fortunately, in the realistic case of strong $J(r)$ and large Zeeman splitting $\omega_{\gamma}$ it is simplified, as has been shown above [eqs. (5.6) and (5.7)]. Obtained formulas predict that in the limit $\eta \gg 1$ the ESP is very large: $\mathcal{P}_{c}(\eta \gg 1)=1 / 4$ and $\mathcal{P}_{*_{c}}(\eta \gg 1)=2 / 9$ for $D-D$ and $T-D$ processes, respectively.

Naturally, all above discussed effects manifest themselves in the rates of ESP generation in corresponding bulk processes as well. We are not going to analyze this manifestation. Such an analysis would require the specification of the process under study, which should, perhaps, be done as applied to particular experiments.

Here we will only restrict ourselves to a general remark on the relation between geminate and bulk ESP. Note, that the ESP can be analyzed in terms of the ratio of the ESP generation $(\mathcal{K})$ and reaction $\left(\mathcal{K}_{r}\right)$ rates: $R_{\mathcal{K}}=\mathcal{K} / \mathcal{K}_{r}=\left(p_{S} p_{R_{\gamma}}\right)^{-1} \mathcal{P}$. Similar relation is valid in the presence of the attractive potential [see eqs. (5.5)]. All these relations show that the analysis of the ESP generation in bulk processes provides the information identical to that obtained by studying the geminate ESP.

\section{CONCLUSIONS}

In this work we have analyzed the specific features of the ESP of charge carriers (electrons and holes) in $D-D$ recombination and $T-D$ quenching in disordered semiconductors. Simple expressions for the ESP, generated in both processes, are derived for two models of stochastic relative motion of particles: cage and free diffusion. Both models predict fairly large ESP in geminate $D-D$ and $T-D$ processes (much larger than the thermal ESP at room temperatures). Especially large ESP is expected in the presence of attraction between particles. Naturally, high efficiency of the ESP generation is predicted in corresponding bulk processes as well.

The mechanism, considered in our work, is based on the assumption that the ESP results from quantum transitions between the terms of the spin Hamiltonian of reacting pairs at short distances $(r \sim d)$ near the term crossing regions (as applied to $D-D$ recombination it is called $S T_{-}$-mechanism ${ }^{11}$ ).

It is worth noting, however, that there is another pair mechanism, well known in the theory of the $D-D$ ESP as $S T_{0}$ mechanism. ${ }^{11}$. It also contributes to the ESP of $D$ particles in the case $\Delta g=\bar{g}_{a}-\bar{g}_{b} \neq 0$ [see eq. (3.9)], but according to the estimations $17,18,20,21$, for typical parameters of systems considered this contribution is much smaller than those considered in our work.

\section{ACKNOWLEDGEMENTS}

The work was partially supported by the Russian Foundation for Basic Research.
1 E. I. Rashba, J. Supercond. 15, 13 (2002).

2 I. Z̆utić, J. Fabian, and S. Das Sarma, Rev. Mod. Phys., 76, 323 (2004).

3 V. A. Dediu, L. E. Hueso, I. Bergenti, and C. Taliani, Nature Mater. 8, 707 (2009).

${ }^{4}$ V. Dediu, M. Murgia, F. C. Matacotta, C. Taliani, and C. Barbanera, Solid State Commun. (London) 122, 181 (2002).

5 Z. Xiong, D. Wu, Z. V. Vardeny, and J. Shi, Nature (London) 427, 821 (2004).

${ }^{6}$ V. N. Prigodin, J. D. Bergeson, D. M. Lincoln, and A. J. Epstein, Synth. Met. 156, 757 (2006).

7 B. $\mathrm{Hu}$ and Y. Wu, Nature (London) 6, 985 (2007).

8 D. R. McCamey, H. A. Siepel, S.-Y. Paik, M. J. Walter, N. J. Borys, J. M. Lupton, and C. Boehme, Nature (London) 7, 723 (2008).

9 P. A. Bobbert, T. D. Nguyen, W. Wagemans, F. W. A. van Oost, B. Koopmans, and M. Wohlgenannt, Synth. Met. 160, 223 (2010).

10 A. J. Schellekens, W. Wagemans, S. P. Kersten, P. A. Bobbert, and B. Koopmans, Phys. Rev. B 84, 075204 (2011).

11 U. E. Steiner and T. Ulrich, Chem. Rev. 89, 51 (1989).

12 C. E. Swenberg and N. E. Geacintov, in Organic Molecular Photophysics, v. 1, edited by J. B. Birks (Wiley, NY, 1973).

13 B. Hu, L. Yan, and M. Shao, Adv. Mater. 21, 1500 (2009).

14 G. L. Closs, J. Am. Chem. Soc. 91, 4552 (1969).
15 R. Kaptein and L. J. Oosterhoff, Chem. Phys. Lett. 4, 195 (1969).

${ }^{16}$ C. Blättler, F. Jent, and H. Paul, Chem. Phys. Lett. 166, 375 (1990).

17 G.-H. Goudsmit, H. Paul, and A. I. Shushin, J. Phys. Chem. 97, 13243 (1993).

18 A. I. Shushin, J. Chem. Phys. 99, 8723 (1993).

19 F. J. Arian, Rev. Chem. Int. 7, 173 (1986).

20 A. I. Shushin, Chem. Phys. Lett. 208, 173 (1993).

21 A. I. Shushin, Chem. Phys. Lett. 260, 261 (1996); Chem. Phys. Lett. 313, 246 (1999).

22 J. H. Freed and J. B. Pedersen, Adv. Magn. Reson. 8, 1 (1976).

23 A. I. Shushin, Chem. Phys. 144, 201 (1990); 144, 223 (1990).

24 A. I. Shushin, Mol. Phys. 58, 101 (1986); J. Chem. Phys. 101, 8747 (1994).

25 A. I. Shushin, Phys. Rev. B 84, 115212 (2011).

${ }^{26}$ K. Schulten and P. G. Wolynes, J. Chem. Phys. 68, 3292 (1978).

27 A. Carrington and A. D. McLachlan, Introduction to magnetic resonance (Harper\&Row Publishers, New York, 1967).

${ }^{28}$ V. Ern and R. E. Merrifield, Phys. Rev. Lett. 21, 609 (1968). 